\title{
SIZING AND DEVELOPMENT OF A PROTOTYPE FOR THE REMOVAL OF WHEELS FROM HEAVY LINE VEHICLES
}

\author{
Anderson Caldas de Oliveira ${ }^{1}$, Danilo Reis da Silva ${ }^{2}$, Jean da Silva de Abreu Silva ${ }^{3}$, Raquel Alves \\ do Nascimento Corrêa ${ }^{4}$, Cledineudo Souza Mendes ${ }^{5}$, Anderson de Oliveira Castro ${ }^{6}$
}

1,2,3,6 Centro Universitário do Norte - UNINORTE - Laureate Universites, Brasil, Rua Leonardo Malcher, 715 - Centro, Manaus/AM.

${ }^{4}$ Instituto de Dados da Amazônia - IDAAM, Brasil, Avenida Djalma Batista. 1719 - Edifício Atlantic Tower, Torre Business, $16^{\circ}$ andar - Chapada, Manaus/AM.

${ }^{5}$ Escola Superior De Tecnologia - EST/UEA, Brasil, Av. Darcy Vargas, 1.200 - Parque Dez de Novembro, Manaus/AM.

Email: anderson.oliiveira@hotmail.com.br,dimooreis@gmail.com,jean.s.abreu@hotmail.com, rakel.an@hotmail.com, ne.udo.sousa@hotmail.com, anderson.castro@uninorte.com.br

Received: May 15th 2019

Accepted: May 24th, 2019

Published: September $30^{\text {th }}$, 2019

Copyright $@ 2016$ by authors and Institute of Technology Galileo of Amazon (ITEGAM). This work is licensed under the Creative Commons Attribution International License (CC BY 4.0). https://creativecommons.org/lice nses/by/4.0/

Open Acteste

\section{ABSTRACT}

With the increase of the fleet of heavy vehicles and the knowledge that tires are one of the components that suffer most from natural wear, inherent to the use of the work routine, the maintenance or replacement of parts and components of the region allocated to the tyre occurs with Often and requires great physical effort. This article focuses on demonstrating the application of the mechanical design of a device that assists in the removal of the wheels of vehicles in this category, as well as to present the equation of the various efforts to which they are submitted, Using specific software such as AutoCAD, Inventor HSM and others. The project elaborated through these projection software in 3D, facilitates the construction and discrimination of the material necessary for the elaboration of the prototype, grounded in the calculations of determination of the static force and application of the safety coefficient, determination of reactions in the support, moment and maximum cutting forces, determination of yield strength in the tube, bearing type determination by the applied effort.

Keywords: Wheels. Mechanism. Device. Sizing.

\section{DIMENSIONAMENTO E DESENVOLVIMENTO DE UM PROTÓTIPO PARA A REMOÇÃO DE RODAS DE VEÍCULOS DA LINHA PESADA}

\section{RESUMO}

Com o aumento da frota de veículos pesados e o conhecimento de que os pneus são um dos componentes que mais sofrem de desgaste natural, inerentes ao uso da rotina de trabalho, a manutenção ou substituição de peças e componentes da região alocada ao pneu ocorre com bastante frequência e requer grande esforço físico. $\mathrm{O}$ presente artigo centra-se em demonstrar a aplicação do projeto mecânico de um dispositivo que auxilia na remoção das rodas de veículos desta categoria, bem como apresentar o equacionamento dos diversos esforços aos quais estão submetidos, utilizando softwares específicos como AutoCAD, Inventores HSM e outros. O projeto, elaborado através destes softwares de projeção em 3D, facilita na construção e discriminação dos materiais necessários para a elaboração do protótipo, com embasamento nos cálculos de reações nos apoios, momento e forças cortantes máximas, determinação do limite de escoamento no tubo, determinação do tipo de rolamento pelo esforço aplicado.

Keywords: Rodas. Mecanismo. Dispositivo. Dimensionamento. 


\section{INTRODUÇÃO}

Tendo em vista veículos da linha pesada e o conhecimento de que pneus são um dos componentes que mais sofrem com o desgaste natural, inerente a utilização da rotina de trabalho, a manutenção ou a substituição de peças e componentes da região alocado o pneu, ou do próprio pneu, sempre há a necessidade de remoção das rodas e seu conjunto para execução dos reparos, o que requer grande esforço físico e tempo. Partindo deste pressuposto, o presente trabalho destinou-se a elaborar um dispositivo para auxiliar na remoção de rodas de veículos da linha pesada, protótipo esse especificamente desenvolvida para remoção de rodas.

Sendo assim, presente trabalho visa desenvolver um dispositivo para auxiliar na remoção de rodas de veículos da linha pesada, haja vista que método atual para execução da manutenção, exige um grande esforço físico do profissional, e de ao menos dois colaboradores, evidenciando o gasto de mão-de-obra e tempo, bem como, à falta de segurança de tal procedimento. Com isso, a manutenção não consegue satisfazer aos requisitos da modernidade, impondo uma necessidade crescente nas mudanças das metodologias e gestão. Assim, a origem do Protótipo visa uma solução prática, utilizando conhecimentos adquiridos durante curso acadêmico de Engenharia Mecânica

A esse processo de reformulação o comércio automotivo no seguimento de manutenção e reparos em veículos de linha pesada, tem passado por uma evolução significativa nas últimas décadas, com mudanças que se desenvolvem através de um maior índice de automação e do uso de equipamentos tecnológicos, buscando sempre inovações em desempenho, custo e qualidade, que tem se tornado armas estratégicas de competitividades e concorrência para que sejam capazes de obter o sucesso nessa categoria. Por outro lado, a manutenção desses veículos deve acompanhar essas melhorias, empregando todas as técnicas e equipamentos disponíveis para um mantimento mecânico moderno [1].

Na busca de desenvolver o protótipo, a criação do modelo em software e a execução do protótipo. Assim como [2], afirmam que os projetos realizados com base em sistemas de auxílio à engenharia não se restringem tão somente ao desenho impresso como também nas informações referentes ao protótipo, levando a uma melhoria na inserção do projetado ao ambiente de execução.

O gerenciamento posto em prática tende a criar algo novo. E o Projeto Mecânico é em suma tudo o que se prevê para o funcionamento de uma máquina ou equipamento. Segundo [3], trata-se de empreendimento complexo, no qual são necessárias diversas ferramentas, que possibilitam avaliar tudo o que se pretende criar, dividindo a complexidade em partes menores, afim de facilitar o progresso, realizando-o em cada etapa, em uma sequência, que abrange todo o escopo do trabalho.

\section{REFERENCIAL TEÓRICO}

Ainda para [3], nos projetos mecânicos existem métodos e materiais padronizados a serem seguidos de forma que criam uma rede de conhecimento e técnicas, incluindo dimensionamento e tolerâncias, unidades e cálculos.

Para a engenharia mecânica, os projetos mecânicos estão na essência do curso, pois pode abranger todas ou várias das disciplinas vistas no curso, desde conhecimento em termodinâmica, para projetos de refratários, como mecânica dos fluidos, para determinar o comportamento dos gases dentro do mesmo refratário. Assim [3], expõem que todas as disciplinas de engenharia mecânica fazem parte de um projeto mecânico direta ou indiretamente.
De acordo com [3], para a determinação em um projeto deve- se levar em conta as seguintes características: Funcionalidade; Resistência/tensão; Desgaste; Segurança; Confiabilidade; Fabricabilidade; Utilidade; Custo; Forma; Tamanho; Mercantilidade.

Fundamental na execução dos projetos, a determinação de cargas e as consequentes reações do objeto fundamentam e norteiam os projetistas às especificações que devem existir para o correto e eficiente funcionamento do produto a ser criado.

Segundo [4], para o projeto de qualquer equipamento em primeiro lugar devem ser considerados os princípios das estáticas para determinar as forças e reações atuantes no projeto, determinando os parâmetros necessários para a escolha dos materiais, geometria e as cargas atuantes nas deflexões e estabilidades do equipamento.

Para [3], define resistência como uma propriedade do material mecânico que depende da escolha, do tratamento e de dados referentes aos processos a qual é submetido o material. Enquanto que para tensão entende-se como a propriedade do estado do material quando este é submetido a uma carga, a depender da geometria, da temperatura e do processo de fabricação.

Para a compreensão mais completa sobre forças atuantes nos corpos e suas consequentes resultantes atreladas aos comportamentos dos corpos [4], determina as seguintes definições: - $\quad$ Resistência dos materiais é um estudo da relação entre as cargas externas que agem sobre um corpo e a intensidade das cargas internas no interior do corpo.

- $\quad$ Forças externas podem ser aplicadas a um corpo como cargas de superfície distribuídas ou concentradas ou como forças de corpo que agem em todo o volume do corpo.

- Cargas distribuídas lineares produzem uma força resultante cujo valor é igual à área sob o diagrama de carga e cuja localização passa pelo centroide dessa área

- $\quad$ Um apoio produz uma força em uma determinada direção sobre o elemento a ele acoplado, se ele impedir a translação do elemento naquela direção e produz momento sobre o elemento se impedir a rotação.

- As equações de equilíbrio $\sum F=0$ e $\sum M=0$ devem ser satisfeitas de modo a impedir, respectivamente, a translação com movimento acelerado e a rotação de um corpo.

- $\quad$ Ao aplicarmos as equações de equilíbrio, é importante desenhar o diagrama de corpo livre antes, de modo a considerar todos os termos presentes nas equações.

- $\quad$ O método das seções é usado para determinar as cargas resultantes internas que agem sobre a superfície do corpo secionado. Em geral, essas resultantes consistem em uma força normal, uma força de cisalhamento, um momento de torção e um momento fletor [4].

Para tensão [4], determina como sendo o quociente entre uma determinada força e um plano a ser estudado, onde a intensidade dessa força entende-se como tensão. Ainda sobre, pode-se determinar sua intensidade pela seguinte fórmula.

Para tensão normal, tal que, a intensidade de força que se aplica em um plano no sentindo perpendicular, segundo [4], dar-se por:

$$
\sigma_{z}=\lim _{\Delta A \rightarrow 0} \frac{\Delta F z}{\Delta A}
$$

Enquanto que, para tensão de cisalhamento onde a intensidade da força se dá tangencialmente em relação ao plano, segundo [4], dar-se por: 


$$
\begin{gathered}
\tau_{z x}=\lim _{\Delta A \rightarrow 0} \frac{\Delta F x}{\Delta A} \\
\tau_{z y}=\lim _{\Delta A \rightarrow 0} \frac{\Delta F y}{\Delta A}
\end{gathered}
$$

Equacionado e obedecendo as premissas de deformação uniforme e constante e considerando um material homogêneo e uniforme pode-se determinar uma tensão média, dada pela equação [4]:

$$
\sigma=\frac{P}{A}
$$

Onde $\sigma$ é a tensão média, $\mathrm{P}$ é força normal e (A) a área que sofre com a carga.

Norton [5], também afirma que para a determinação de tensão para perfis específicos, deve-se utilizar a equação:

$$
\sigma=\frac{M * c}{I}
$$

Onde $\mathrm{M}$ é o momento fletor, $\mathrm{C}$ a distância do centroide da peça e I seu momento de inercia.

A figura 1 exemplifica a aplicação da tensão de cisalhamento
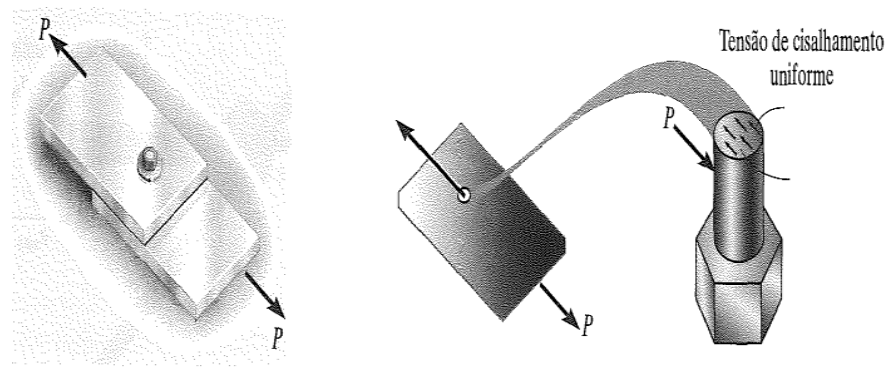

Figura 1: Aplicação de tensão de cisalhamento.

Fonte: [4].

\section{II.1 DIAGRAMA TENSÃO X DEFORMAÇÃO}

Segundo [5] o diagrama tensão $X$ deformação figura 5 fornece diversas informações necessárias para a compreensão do comportamento das estruturas quando acometidas de esforços. Nele pode-se avaliar a tensão em proporcionalidade com a deformação, expressa na lei de Hooke. E o limite de proporcionalidade, ou módulo de elasticidade, chamado também de módulo de Young $(E)$ Com esse parâmetro, pode-se obter o alongamento específico do material pela equação:

$$
\begin{gathered}
E=\frac{L-L_{0}}{L_{0}} \\
E=\frac{\sigma}{\varepsilon}
\end{gathered}
$$

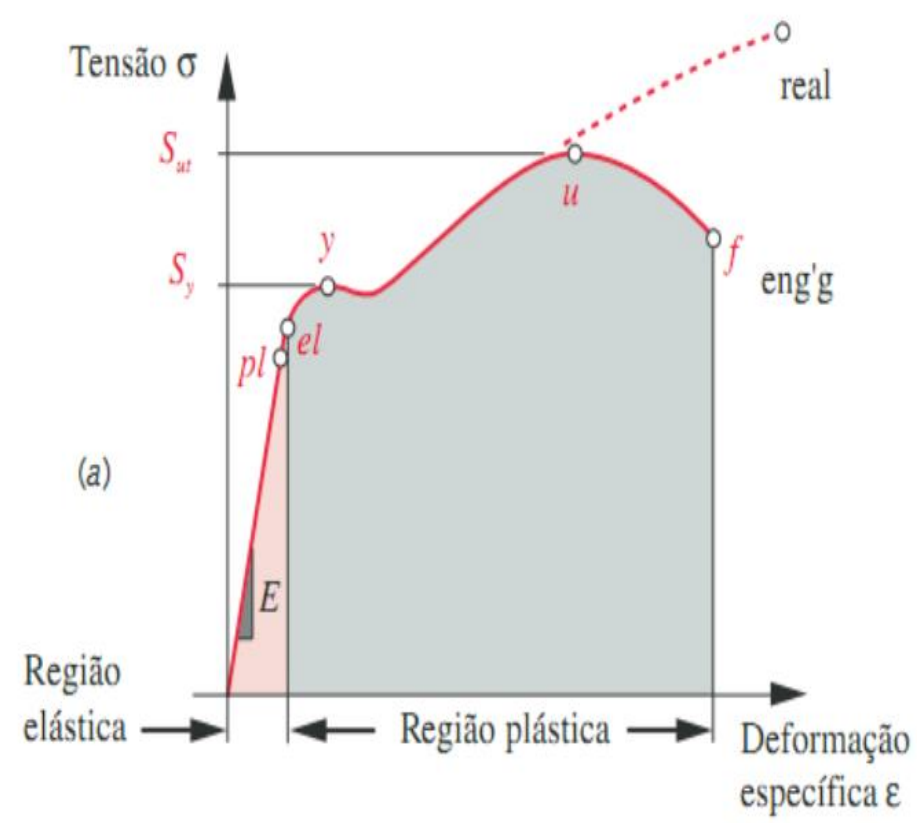

Figura 2: Diagrama tensão X deformação. Fonte: [5].

\section{2 FATOR DE SEGURANÇA}

Com o intuito de possibilitar o desenvolvimento eficaz e seguro de um equipamento, faz-se necessário determinar parâmetros mínimos afim de assegurar a estabilidade e confiabilidade do projeto.

Conforme [6], define o fator de segurança como sendo um número empírico pelo qual a resistência do material é dividida de forma a obter-se um valor conservador de tensão.

Conforme [3], o método determinístico para equalização de tensão máxima admissível, ao final do levantamento de todas as cargas atuantes no equipamento e a tensão máxima do material utilizado, é dado pelo fator de projeto, através da formula:

$$
N d=\frac{P}{\sigma \operatorname{Max}}
$$

Onde (Nd) é baseado na aplicação em que se submeterá o equipamento e o material a ser utilizado.

Enquanto [4], considera fator de segurança a relação entre a força corrigida e a força aplicada, na fórmula:

$$
F_{S}=\frac{\text { Fcorrigida }}{F}
$$

Onde,

$F_{S=}$ é o fator de segurança aplicado segurança

Fcorrigida $=$ é a força após a aplicação do fator de

$F=$ é a força originalmente exercida

Para uma análise acurada e perspicaz, [7] recomenda a conforme tabela 01 .

Onde $E=$ Modulo de elasticidade

$\varepsilon=$ Deformação específica

$\sigma=$ Tensão em Pascal

$L=$ Comprimento final

$L_{0=}$ Comprimento inicial 
Tabela 1: Fatores de seguranças recomendados.

\begin{tabular}{|c|c|}
\hline $\begin{array}{c}\text { Fator de } \\
\text { Segurança }\end{array}$ & Recomendações \\
\hline 1,25 a 1,5 & $\begin{array}{l}\text { Para materiais excepcionalmente } \\
\text { confiáveis a serem utilizados sob } \\
\text { condições controladas e sujeitas a } \\
\text { cargas e tensão que possam ser } \\
\text { determinadas com alta gral de } \\
\text { precisão, utilizados quase que } \\
\text { invariavelmente onde o baixo peso é } \\
\text { uma consideração particularmente } \\
\text { importante. }\end{array}$ \\
\hline 1,5 a 2 & $\begin{array}{l}\text { Para materiais bem conhecidos, sob } \\
\text { condições ambientais razoavelmente } \\
\text { constante, sujeitas a carga e tensões } \\
\text { que podem ser determinadas } \\
\text { facilmente. }\end{array}$ \\
\hline 2 a 2,5 & $\begin{array}{l}\text { Para materiais cujas propriedade } \\
\text { sejam conhecidas em termos de } \\
\text { médias, operados em ambiente } \\
\text { comuns e sujeitos a cargas e tensão } \\
\text { que possa ser determinada. }\end{array}$ \\
\hline 2,5 a 3 & $\begin{array}{c}\text { Para materiais pouco testado ou } \\
\text { frágeis sujeita a condições ambientais, } \\
\text { carga e tensões. }\end{array}$ \\
\hline 3 a 4 & $\begin{array}{l}\text { Podem ser utilizados para materiais } \\
\text { cujas propriedade sejam bem } \\
\text { conhecidas e que devem ser utilizadas } \\
\text { em ambiente incertos ou sujeito a } \\
\text { tensões incertas. }\end{array}$ \\
\hline
\end{tabular}

A) Cargas repetidas: os fatores estabelecidos nos itens 1 e 6 são aceitáveis, porem devem ser aplicados ao limite de resistência a fadigas, em vez de a resistência ao escoamento do material.

B) Força de impacto: os fatores fornecidos nos itens 3 a 6 são aceitáveis, porém um fator de impacto deve ser incluído no projeto.

C) Materiais frágeis: nos casos em que o limite de resistência é utilizado como valor máximo teórico, os fatores apresentados nos itens 1 a 6 devem ser aproximadamente dobrados.

D) Nos casos em que fatores mais altos possam parecer mais apropriados, uma análise mais detalhada do problema deve ser realizada antes da decisão sobre o valor desses fatores.

Fonte: Adaptado de [7].

\section{II.3 FIXAÇÃO DE ELEMENTOS POR SOLDA}

Há muito já se fabricava máquinas e equipamentos totalmente em ferro fundido cinzento, que apresenta um bom amortecimento. No entanto o emprego do processo de soldagem possibilitou a fabricação e peças em aço com partes soldadas, mesmo que acabadas em processo de usinagem, com valores de resistência maiores e pesos menores em relação ao ferro fundido cinzento [5].

A soldagem, soldadura ou solda, é o processo de união entre duas ou mais peças pela fusão, a nível molecular, na superfície das partes soldadas [8].

No processo de soldagem algumas definições são importantes, como a escória, que trata-se do fluxo de material deixado durante a soldadura afim de evitar a contaminação do cordão de solda pela humidade e o ar do ambiente. Já o cordão de solda é a massa resultante da combinação do metal fundido entre o material de adição e a material base. Durante esse processo, na material base, ocorre a ZTM, Zona Termicamente Afetada, sendo a região onde a temperatura no processo de solda afetará as propriedades da material base, normalmente próximo ao cordão de solda [5].

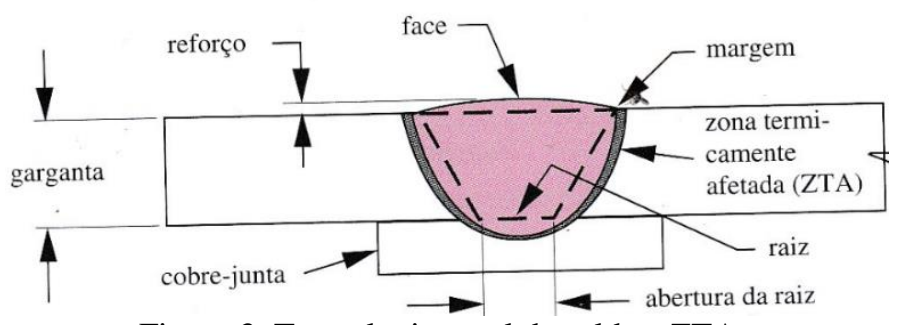

Figura 3: Termologia geral da solda e ZTA.

Fonte: [7].

Norton [5] define que no processo de soldagem existem diversos tipos de máquinas e técnicas adequadas para cada atividade planejada com soldagem. Tais como:

- Soldagem com eletrodos revestidos:

- Soldagem com arame tubular;

- Soldagem a arco gás-metal;

- Soldagem a arco gás-tugstênio;

- Soldagem ao arco submerso;

- Soldagem por resistência.

Comumente utilizado na indústria os tipos de solda eletrodo revestido e arame tubular representam a maior parte dos processos utilizados, juntamente com a soldagem arco gás para serviços mais precisos e com acabamentos superiores.

Para a soldagem com eletrodos revestidos, Norton [5] define como a solda na qual é utilizada varetas de eletrodos com tamanhos definidos com revestimento com a função de revestir o metal fundido e protege-lo ao final do processo.

O tipo de solda arame tubular utiliza um eletrodo na forma tubular que permite a passagem do fluido de gás por seu interior, tornando o processo automático e rápido, o que possibilita a execução de longos tubos de solda. A máquina de solda pode ser adequada conforme necessidade do operador [5].

Também conhecida como solda MIG (metal inert gás), o processo de soldagem arco gás-metal, usa um eletrodo de arame sem fluxo, e o gás inerte é dirigido para a região da solda afim de expulsar o ar evitando a contaminação [5].

$\mathrm{Na}$ união de das peças são aplicadas técnicas distintas quanto à necessidade e adaptabilidade do local de solda. Assim, segundo Norton [5], há seis tipos principais de tipos de juntas: topo, tê, canto, sobreposta e aresta, a ser definida principalmente pela geometria da peça solda. Essas juntas podem ser vistas na figura 4.

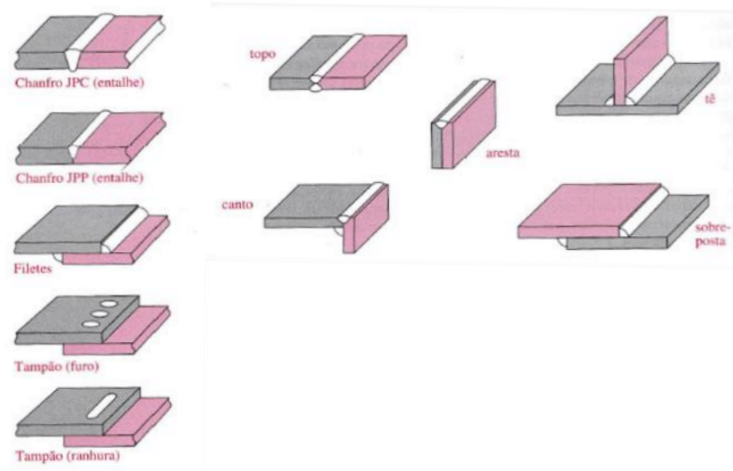

Figura 4: principais de tipos de juntas. Fonte: [5]. 
Para Norton [5], as uniões em chanfro podem ser juntas de penetração completa (JPC) ou junta de penetração parcial (JPP). Esses tipos de uniões, juntamente com a junta filete são representados na figura 5 .

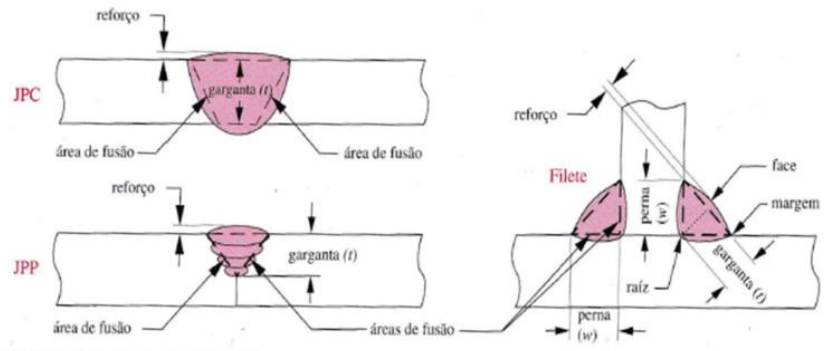

Figura 5: juntas JPC, JPP e filete. Fonte: [7].

\section{II.4 CARREGAMENTO ESTÁTICO EM SOLDAS}

Devido às variadas formas de junção, o cordão de solda poderá está sujeito a diversas formas de carregamento, no qual a posição do cordão definirá o tipo esforço [8].

Para soldada com junção tipo entalhe JPC, [3], afirma que para o carregamento de tração ou compressão, a tensão normal média e dada pela fórmula:

$$
\sigma=\frac{F}{h l}
$$

solda.

Em que $\boldsymbol{h}$ é a garganta de solda e $\boldsymbol{l}$ o comprimento de

Segundo Pareto [8], a tensão admissível nos cordões de solda é menor que às dos materiais soldadas, mesmo que o material adicionado seja o mesmo da peça. Assim a determinação das tensões admissíveis dependerá do tipo de solda e do material soldado, como visto na tabela 2.

Tabela 2: Resistência de cordão de solda Tabela.

\begin{tabular}{|c|c|c|}
\hline Tipo de solda & $\begin{array}{c}\text { Classe de } \\
\text { esforço }\end{array}$ & $\begin{array}{c}\text { Resistência } \\
\text { da solda }\end{array}$ \\
\hline \multirow{3}{*}{ De topo } & Tração & $0,85 \mathrm{k}$ \\
\cline { 2 - 3 } & Compressão & $0,80 \mathrm{k}$ \\
\cline { 2 - 3 } & Flexão & $0,80 \mathrm{k}$ \\
\cline { 2 - 3 } & Torção & $0,65 \mathrm{k}$ \\
\cline { 2 - 3 } & Cisalhamento & $0,65 \mathrm{k}$ \\
\hline De canto & Todos & $0,65 \mathrm{k}$ \\
\hline \multicolumn{2}{|c|}{ Obs. onde k representa a tensão admissível } \\
do dito material \\
\hline \multicolumn{2}{|c|}{ Fonte: [8]. } \\
\hline
\end{tabular}

\section{METODOLOGIA}

No que se refere aos métodos e técnicas empregados na pesquisa, tendo em vista que o direcionamento do trabalho para o desenvolvimento de um dispositivo para a melhoria de um processo e a comparação desse dispositivo com um análogo encontrado no mercado utilizou-se o método exploratório, no qual, [10], envolvem levantamento bibliográfico, entrevista com pessoas que tiveram experiência com o problema de pesquisa.

Neste sentindo, realizou-se inicialmente a pesquisa bibliográfica, afim de fundamentar os conhecimentos em relação aos assuntos inerentes a pesquisa, encontrados em livros, revistas, periódicos, monografias, teses e internet, disponível ao público em geral, que abordaram assuntos tais como, gerenciamento de projetos, elementos de máquinas, ergonomia e história e manutenção de pneus.

A coleta de dados deu-se através da retirada de medidas e acompanhamento do processo de remoção de pneus, o que originou nas medidas necessária para a criação do protótipo, e fomentou dados para o memorial de cálculos com valor da carga de trabalho, relacionado com o dimensional máximo do cubo de roda do pneu, que fornece, através, da aplicação da Segunda Lei de Newton, as forças resultantes no protótipo, que possibilitam a determinação das reações mecânicas na estrutura do carro, como flexão pura, tensão e deformação, conforme [3] e [4], além de técnicas e cálculos de resistência em solda e rolamentos segundo Norton [5] e Pareto [8], findando com o dimensionamento levando em consideração as recomendações de [7] quanto ao coeficiente de segurança para o projeto.

\section{III.1MEMORIAL DE CÁLCULO}

Nessa etapa dimensiona-se matematicamente o protótipo afim de construí-lo dentro das especificações que atendam os objetivos de segurança, de praticidades e financeiro. Dessa forma, para o equacionamento do projeto fora levado em consideração alguns parâmetros iniciais, de construção e carga, tais como:

1. A carga máxima transportada pelo protótipo deve ser de $400 \mathrm{~kg}$

2. A estrutura deve ser feita, principalmente, de tubo comercial;

3. As dimensões dos tubos devem ser suficientes para abraçar o cubo com as duas rodas montadas;

4. O coeficiente de segurança utilizado é igual a 2;

5. O material do tubo é o ASMT A53 schedule 40;

6. Resistencia a tração do material Rt $330 \mathrm{Mpa}$;

7. Limite de escoamento Le é igual a $205 \mathrm{Mpa}$;

8. O conjunto cubo mais pneus é tomado como uma carga uniformemente distribuída;

9. O peso líquido do projeto é de $70 \mathrm{~kg}$;

10. Vida útil do rolamento de 24000 horas;

11. A distância da base do projeto e o piso não devem ser inferiores a $25 \mathrm{~mm}$;

\section{III.2 CRIAÇÃO DO PROTÓTIPO COM AUTODESK INVENTOR HSM}

Uma questão importante na produção de protótipos é a conferência dos modelos virtuais e físicos afim de efetuar os ajustes necessários [9].

No desenvolvimento de protótipos mecânicos é de suma importância a modelagem do mecanismo em 3D, pois é possível observar uma prévia quanto ao design, estrutura e funcionalidade do protótipo. Como ponto inicial para a criação do projeto foi elaborado através de software Inventor hsm uma projeção em 3D do projeto inicial, no qual norteou a construção do protótipo e facilitou a discriminação do material necessário para execução do projeto. Apresentação da perspectiva do projeto em 2D conforme figura 6. 


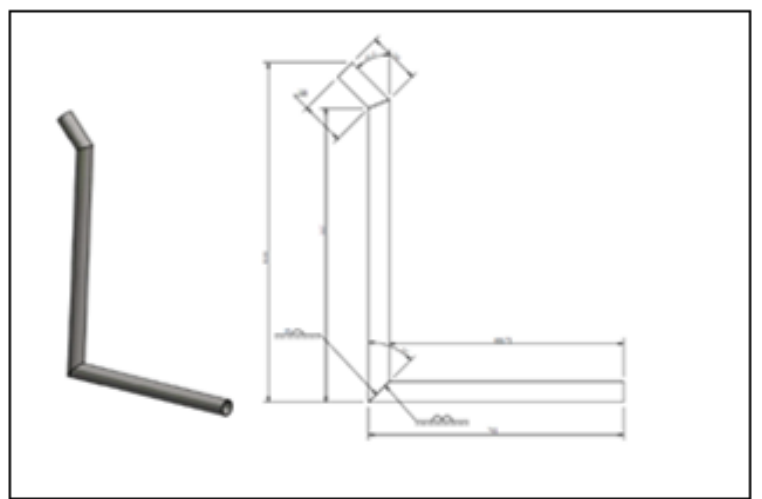

Figura 6: Perspectiva 2D da estrutura externa do projeto. Fonte: Autores, (2019).

Para o desenvolvimento do Projeto, o projeto foi subdividido em três estruturas que fundamentam o funcionamento do dispositivo.

Inicialmente se desenvolveu a estrutura externa do carro, no qual será feita a solda da parte externa do projeto juntamente com a montagem, como pode ser visto na figura 7 .

Figura 7: Estrutura externa do projeto montada e com pontos de solda.

A segunda parte a ser projetada foi à estrutura interna do Projeto. Essa estrutura é articulada para facilitar a regulagem de altura para adaptação às limitações encontradas em cada caso que poderá ser aplicado, como pode ser visto nas figuras 8 e 9 .

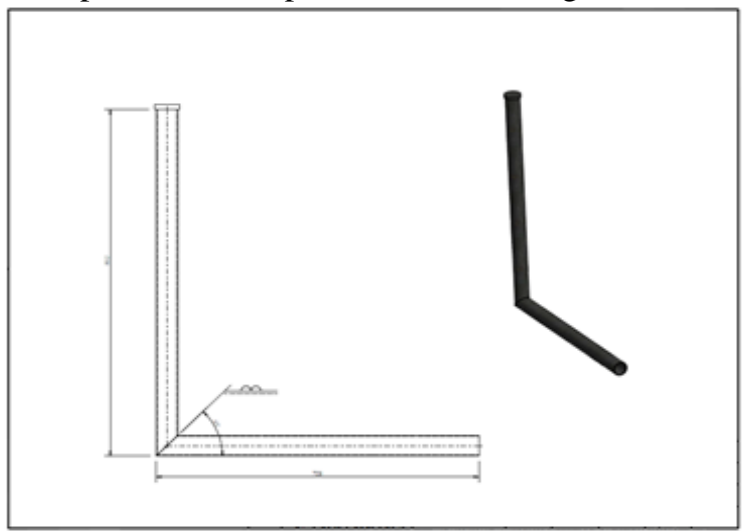

Figura 8: Parte da Estrutura interna móvel Fonte: Autores, (2019).

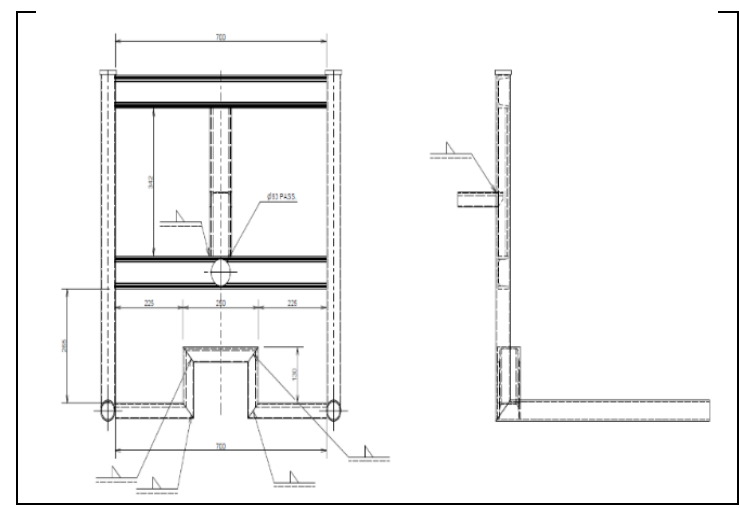

Figura 9: Detalhe da estrutura interna montada e com pontos de solda.

Fonte: Autores, (2019).
A terceira se projeta avalanca, trata-se do mecanismo para facilitar na remoção do pneu instalado no veículo. Para isso, utilisou-se uma estrutura em forma de alavanca, que apoiada ao a estrutura externa, possibilita o emprego de força concentrada na roda, imprimindo movimento movimento a conjunto do cubo ou pneu, executando assim sua retirada. Conforme apresentado na figura 10.

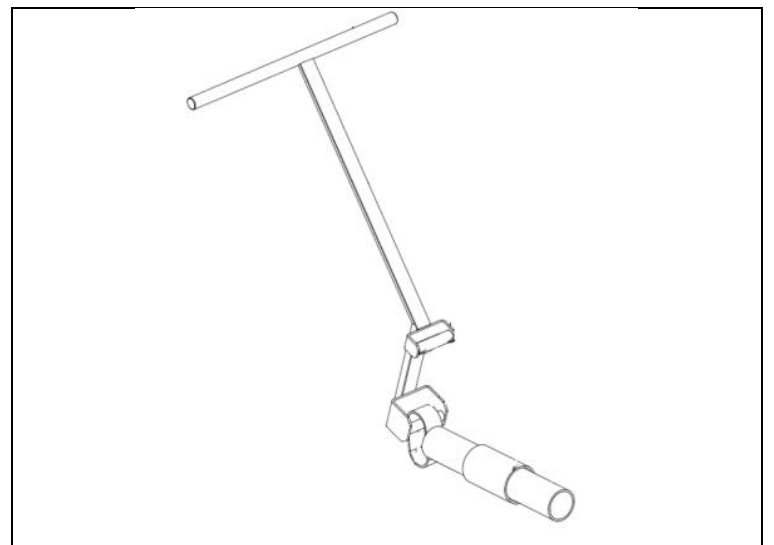

Figura 10: Alavanca de extração.

Fonte: Autores, (2019).

A quanta parte e ultima estrutura do mecanismo finalizada com todos os componetes montado da estrutura, conforme apresentado na figura 11 .

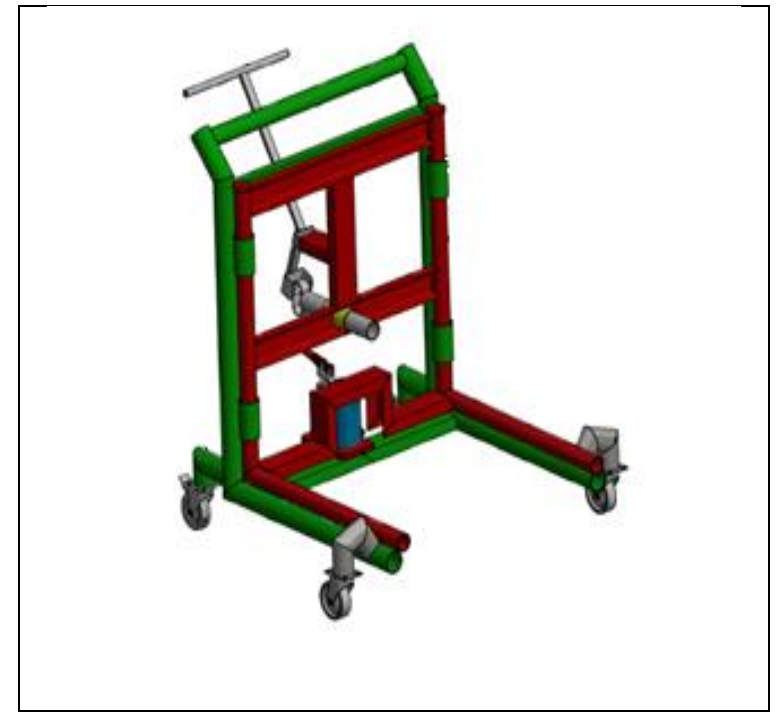

Figura 11: montado com todos os componentes do projeto. Fonte: Autores, (2019).

\section{III.3 DETERMINAÇÃO DA FORÇA ESTÁTICA E APLICAÇÃO DO COEFICIENTE DE SEGURANÇA}

Utilizando a segunda lei de Newton para a determinação da força em função da massa tem-se, segundo [4]:

$$
F=m * a
$$

Onde;

$\mathrm{F}=$ força em $\mathrm{N}$

$\mathrm{m}=$ massa em $\mathrm{Kg}$

$\mathrm{a}=$ aceleração $\mathrm{m} / \mathrm{s}^{2}$ 
Para a resolução do problema adotou-se a aceleração gravitacional igual a $9,81 \mathrm{~m} / \mathrm{s}^{2}$.

Dados: $m=400 \mathrm{~kg}-$ Peso do conjunto da roda, pneu, cubo e tambor.

$F=400 \mathrm{Kg} * 9,81 \mathrm{~m} / \mathrm{s}^{2}$

$$
F=3,924 K N
$$

Aplicando-se o fator de segurança de segurança igual a 2, tem-se:

$$
F_{S}=\frac{\text { Fcorrigida }}{F}
$$

Fcorrigida $=2 * 3,924 K N$

Fcorrigida $=7,84 K N$

\section{III.4 DETERMINAÇÃO DE REAÇÕES NOS APOIOS, MOMENTO E FORÇAS CORTANTES MÁXIMAS.}

Para a determinação de reações nos apoios da estrutura externa horizontal onde serão instalados os rolamentos, que por sua vez são os apoios de todo o projeto, toma-se como referência o esquema visto na figura 12, realizado através do software BeamDesing:

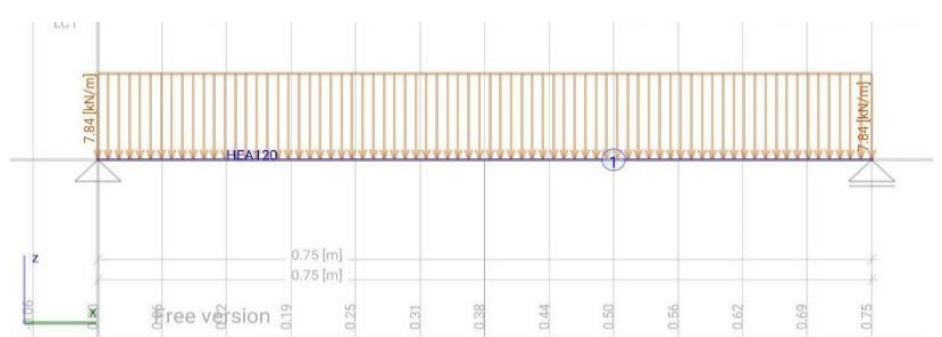

Figura 12: Esquema de forças e apoios para estrutura. Fonte: Autores, (2019).

Ainda com a utilização do software, pode-se visualizar as resultantes nos apoios, os diagramas de momento (M) e o diagrama de força cortante (V), como visto na figura 13.

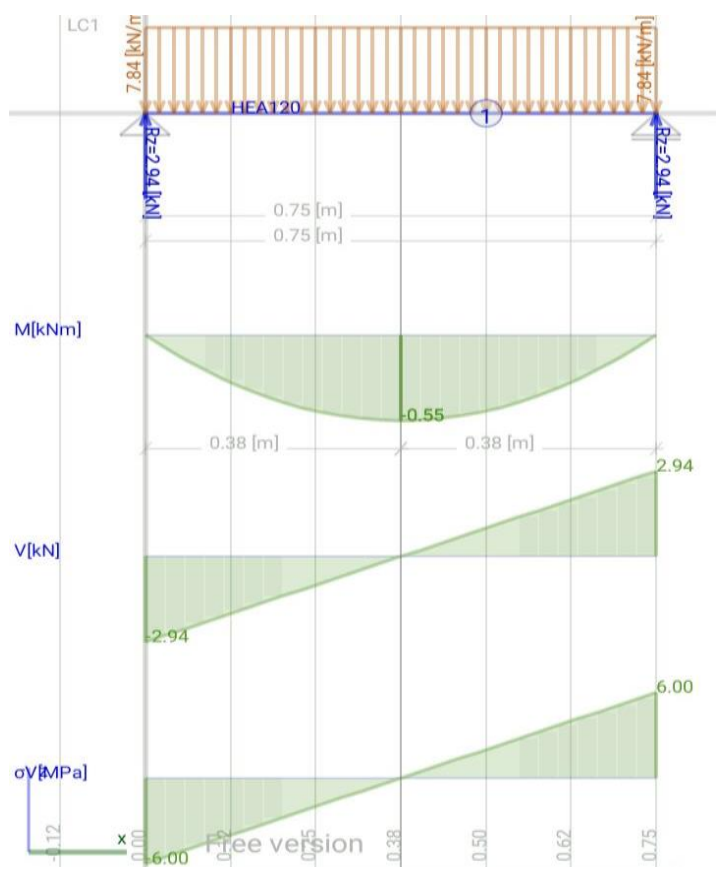

Figura 13: Diagramas de momento, força cortante e reações nos apoios.

Fonte: Autores, (2019).
Esses resultados também podem ser observados nos cálculos de reações de apoio, momento e força cortante, através das fórmulas, como cita [4].

$$
\begin{gathered}
\Sigma f x=0 \\
\Sigma f y=0 \\
\Sigma f M a=0
\end{gathered}
$$

Assim;

$$
\Sigma f y=R A+R B-F * d
$$

$$
\begin{gathered}
R A+R B=7,84 K N * 0,75 \mathrm{~m} \\
R A+R B=7,84 K N * 0,75 \mathrm{~m} \\
R A+R B=5,88 K N \\
\Sigma f M a=0 \\
D f M a=R B * d-5,88 K N * d \\
0=R B * 0,75-5,88 K N * 0.375
\end{gathered}
$$

$\mathrm{RB}=2,94 \mathrm{KN}, \log \mathrm{OA}=2,94 \mathrm{KN}$

Para cálculo da força cortante máxima, segundo [4], podese fazer uso da equação:

$$
\mathrm{V}=\int-\mathrm{y} * \mathrm{dx}
$$

$\mathrm{V}=\int-7,84 * \mathrm{dx}$

$\mathrm{V}=-7,84 \mathrm{x}+\mathrm{C}$

Para a determinação de $\mathrm{C}$ toma-se $\mathrm{x}$ igual a 0 (zero) e V igual RA, logo:

\section{$\mathrm{C}=2,94 \mathrm{KN}$}

Determinando assim a equação para a determinação das foças cortantes, dado por:

$$
V=-7,84 x+2,94
$$

Ainda segundo [4], podemos determinar os momentos na estrutura integrando a força cortante, tal que.

$$
\mathrm{M}=\int \mathrm{Vdx}
$$

$$
\begin{aligned}
& M=\int-7,84 x+2,94 \\
& M=-3,92 x^{2}+2,94 x+C
\end{aligned}
$$

Assim como na equação de força cortante, para a determinação de (C), toma-se (x) igual a 0 (zero) (Ma) também igual a 0 (zero) como visto na somatória de momentos. Assim, a equação para a determinação de momento na estrutura é:

$$
\begin{aligned}
& M=-3,92 x^{2}+2,94 x \\
& \text { Sendo } M m a x=0,551 N * m
\end{aligned}
$$

Determinação da tensão de cisalhamento do tubo da estrutura. Como se propôs utilizar tubos para a construção do projeto, é, portanto, preciso considerar o perfil transversal do tubo para a determinação das tensões de cisalhamento.

Para [4], a tensão plena pode ser obtida pela equação:

$$
\sigma=\frac{P}{A}
$$


Considerando a força obtida nos cálculos anteriores e a tensão máxima para tubos ASMT A53, pode-se determinar a diâmetro externo do tubo, pela equação a seguir.

$$
330 \mathrm{MPa}=\frac{7,84 K N}{\pi \mathrm{R}^{2}}
$$

$\mathrm{R}^{2}=\frac{330 M P a}{7,84 K N \pi}$

$\mathrm{R}=27,49 \mathrm{~mm}$

$\mathrm{D}=54,999 \mathrm{~mm}$

Para tubo comercial mais próximos fora adotado o tubo com diâmetros externo de 2" (polegadas).

Contudo, para a determinação da tensão sobre o tubo, tomando nota o perfil vazado do mesmo, e já conhecendo seu diâmetro externo, aplica-se a seguinte equação:

$$
\sigma=\frac{M * C}{I}
$$

Onde $I$ pode ser determinado por: $I=\frac{\pi}{64} *\left(\left(D^{2}\right)^{2}-\left(d^{2}\right)^{2}\right)$, em que $\mathrm{D}$ equivale a $60,3 \mathrm{~mm}$ e $\mathrm{d} 52,48 \mathrm{~mm}$, tomando como base a Norma Brasileira NBR 5590 para tubos schedule 40.

Assim,

$$
\sigma=\frac{0,55 * 0,0635}{2.7664 * 10^{\wedge}-7}
$$

$\sigma=126, G \mathrm{~Pa}$

\section{III.5 DETERMINAÇÃO DO LIMITE DE ESCOAMENTO NO TUBO}

Como última análise para a estrutura externa do tubo, fora calculado o limite de escoamento no material ao ser exposto a tensão computada, tomando como equação o módulo de Young, tal como:

$$
\begin{aligned}
& E=\frac{\sigma}{\varepsilon} \\
& 2050=\frac{126}{\varepsilon} \\
& \varepsilon=0,061
\end{aligned}
$$

Para determinar o alongamento do tubo fora utilizado a equação da deformação específica.

$$
\varepsilon=\frac{L-L_{0}}{L_{0}}
$$

$$
\begin{aligned}
& \begin{array}{l}
0,061=\frac{L-0,75}{0,75} \\
\mathrm{~L}=0,8 \mathrm{~mm}
\end{array} \\
& \text { III.6 DETERMINAÇÃO DO TIPO DE ROLAMENTO PELO } \\
& \text { ESFORÇO APLICADO }
\end{aligned}
$$

\section{III.6 DETERMINAÇÃO DO TIPO DE ROLAMENTO PELO ESFORÇO APLICADO}

Para uma escolha fundamentada e precisa dos rolamentos, que são os suportes do projeto, foram determinadas peças que atendassem aos requisitos de diâmetro, valor de mercado e capacidade de carregamento. Essa avaliação pode ser realizada a partir da determinação dos esforços nos apoios que norteou a eleição dos rolamentos com base em suas tabelas técnicas. Como o esforço máximo aplicado em cada rolamento é de 2,94 KN, ou seja,
$294 \mathrm{Kg}$ pode-se determinar a carga máxima aplicada ao rolamento, pela equação:

$$
\begin{gathered}
F=\frac{2 F M+F m}{3} \\
F=\frac{2 * 2,94 K N+7000 N}{3} \\
F=4.3 K N \\
F=430 k g \\
C=\frac{f h * F}{f n} \\
C=\frac{3,5 * 4300 N}{1,5} \\
C=10000 N
\end{gathered}
$$

Desta forma, o rolamento comercial que atendeu os requisitos impostos pelas dimensões do carro e ao mesmo tempo suporta as condições de carga e utilização, com base nos dados técnicos do fabricante, fora o rolamento fixo de uma carreira 6910 ZZ VV DDU.

\section{III.7 DETERMINAÇÃO DAS TENSÕES NOS PONTOS DE SOLDA}

Para a determinação das resistências às tensões atuantes nos pontos soldados, tomou-se como referência a tabela 2 , deste trabalho, que expõe a tensão nos pontos de soldas, para solda de canto, utilizada na construção do projeto, é dada como $0,65 \mathrm{~K}$. Dessa maneira, a tensão admissível nos pontos de solda, em relação ao material de construção equivale a $210 \mathrm{MPa}$. Valor que se enquadra nos esforços já calculados.

\section{CONCLUSÃO}

Sempre que pensamos em projetar/criar uma peça ou equipamento, precisamos ter em mente as diversas formas de representar as nossas ideias. O projeto mecânico, visa a melhor forma de representar através do desenhos técnicos, que especificam todas as medidas e formas dos objetos, e têm a garantia de serem interpretados de forma clara, assim o detalhamento de Projeto são feitos em softwares CAD, como AutoCad, Inventor HMS e Outros. projeto, é necessário que haja a garantia de funcionalidade por etapas de Cálculos Estruturais como demostrado nos tópicos : III.3 Determinação da força estática e aplicação do coeficiente de segurança, III.4 Determinação de reações nos apoios, momento e forças cortantes máximas,III.5 Determinação do limite de escoamento no tubo, III.6 Determinação do tipo de rolamento pelo esforço aplicado. Onde estabelece-se a dimensão e a capacidade de sustentação dos elementos básicos de uma estrutura, onde são dimensionados os componentes, e é feito com embasamento nos cálculos. O projeto desenvolvido baseia-se no esforço manual do operador, desta forma, é possível adaptar dispositivo pneumático ou hidráulico visando reduzir o esforço físico do operador.

\section{REFERÊNCIAS}

[1] Fleming, Paulo V. França Sandro R.R.O.; Considerações Sobre a implementação Conjunta de TPM e MCC na Indústria de Processos; ABRAMAN; 1997. 
[2] Panizza, A.C.; Ruschel, R.C. Avaliação da Competência Para Colaboração em CAD Associada à Utilização da Tecnologia da Informação no Desenvolvimento do Projeto. In: Seminário de Tecnologia da Informação e Comunicação na Construção Civil, 2., 2005, São Paulo., 2005. Disponível em: <www.infohab.org.br/acervos/buscatipo/codigoTipo/10/page/235 3> acesso em: 14 de ago. 2017.

[3] Budynas,Richard; Elemento de máquinas de Shigley Projeto de engenharia mecânica . Tradução técnica João Batista deAguiar, 8. ed. Porto Alegre, 2011.

[4] Hibbeler, Russell Charles Resistência dos materiais I Russell Charles Hibbeler 7. ed. - São Paulo : Pearson Prentice Hall, 2010.

[5] Norton, Robert L., Projetosde Máquinas: uma abordagem integrada . ed. - Porto Alegre, Bookman, 2013.

[5]Robert L. Norton -Projeto de máquinas. $4^{\circ}$ ed. Porto Alegre. Bookman. 2013.

[6] Chiaverini, Vicente. Tecnologia mecânica: estrutura e propriedades das ligas metálicas. Volume I. 2. ed. São Paulo: McGraw-Hill, 1986.

[7] Juvinall, R C; Marshek, K. M. Fundamento do projeto de componentes de máquina.4.ed. Rio de Janeiro: LTC, 2008.

[8] Pareto, Luis, Formulário técnico elementos de máquinas, CEAC S.A, Barcelona (Espanha), 2003, por Hemus.

[9]Grimm, Todd. Rapid Prototyping - User's Guide. Society of Manufacturing Engeneers, 2004

[10] Gil, Carlos Antonio, 1946 Como elaborar Projetos de Pesquisa -3 ed.- São Paulo: Atlas 1991. 